\title{
Two-State Membrane Potential Transitions of Striatal Spiny Neurons as Evidenced by Numerical Simulations and Electrophysiological Recordings in Awake Monkeys
}

\author{
Katsunori Kitano, ${ }^{1}$ Hideyuki Câteau, ${ }^{1,2}$ Katsuyuki Kaneda,, ${ }^{2,3}$ Atsushi Nambu, ${ }^{2,3}$ Masahiko Takada,, ${ }^{2,3}$ and \\ Tomoki Fukai ${ }^{1,2}$ \\ ${ }^{1}$ Department of Information-Communication Engineering, Tamagawa University, Machida, Tokyo 194-8610, Japan, ${ }^{2}$ Core \\ Research for Evolutional Science and Technology, Japan Science and Technology Corporation, Kawaguchi, Saitama \\ 332-0012, Japan, and 3Department of System Neuroscience, Tokyo Metropolitan Institute for Neuroscience, Tokyo \\ Metropolitan Organization for Medical Research, Fuchu, Tokyo 183-8526, Japan
}

\begin{abstract}
Spontaneous membrane potential fluctuations of striatal spiny projection neurons play a crucial role in their spike generation. Previous intracellular recording studies in anesthetized rats have shown that the membrane potential of striatal spiny neurons shifts between the depolarized "up" state and the hyperpolarized "down" state. Here we report evidence for the occurrence of such two-state membrane potential transitions by numerical simulations and electrophysiological recordings in awake monkeys. Data from our simulations of a striatal spiny neuron model demonstrated that spike latency histograms of the model neuron displayed two separate (i.e., early and late) peaks in response to excitatory cortical input, corresponding to neuronal activity in the up or down state, respectively. Then, we addressed experimentally whether the latency distribution of
\end{abstract}

The basal ganglia are a group of subcortical structures that are interconnected with one another to participate in various aspects of motor behavior (Graybiel et al., 1994; Graybiel, 1995). The striatum is a main input station of the basal ganglia and receives diverse inputs from widespread areas of the cerebral cortex (Parent and Hazrati, 1995). Medium-sized spiny neurons constitute populations of projection neurons in the striatum. According to a series of in vivo intracellular recording studies by Wilson and his colleagues (Wilson and Groves 1981; Wilson, 1993; Wilson and Kawaguchi, 1996; Stern et al., 1997, 1998; Wickens and Wilson, 1998), the membrane potential of striatal spiny projection neurons in urethane-anesthetized rats spontaneously repeats fluctuations between two subthreshold levels, the depolarized "up" state and the hyperpolarized "down" state. These two states of the membrane potential are separated by $15-30 \mathrm{mV}$, and the mean potential of the up state is usually $3-5 \mathrm{mV}$ below spike threshold. Therefore, up transitions from the down to the up state seem critical for spike firing in striatal spiny neurons, a majority of which are silent without spontaneous firing. Several lines of evidence indicate that such membrane potential state transitions are yielded by excitatory input from the cortex (Wilson et al., 1983; Kawagu-

\footnotetext{
Received Dec. 14, 2001; revised March 11, 2002; accepted March 29, 2002.

K.K. is supported by Japan Society for the Promotion of Science.

Correspondence should be addressed to Dr. Tomoki Fukai, Department of Information-Communication Engineering, Tamagawa University, 6-1-1 Tamagawagakuen, Machida, Tokyo 194-8610, Japan. E-mail: tfukai@eng.tamagawa.ac.jp. Copyright (C) 2002 Society for Neuroscience $0270-6474 / 02 / 220001-\bullet \$ 15.00 / 0$
}

cortically induced spike firing of striatal spiny neurons might show dual peaks. Striatal neuron activity was extracellularly recorded in response to electrical stimulation in the two cortical motor-related areas, the primary motor cortex and the supplementary motor area, of awake monkeys. Analysis of spike latency histograms has defined that striatal spiny neurons typically exhibit two temporally distinct peaks, as obtained by the numerical simulations. Thus, the membrane potential shifts between the up and down states appear to occur in striatal spiny neurons of the behaving animal.

Key words: striatal spiny projection neuron; corticostriatal input; multicompartment neuron model; extracellular recording; spike latency histogram; awake monkey chi et al., 1989). Cortical stimulation has indeed been shown to elicit depolarizing phenomena in the striatum that well resemble the up transitions occurring spontaneously (Wilson, 1993, 1995; Wilson and Kawaguchi, 1996). In the present study, we attempted to analyze the cortically induced two-state membrane potential transitions by numerical simulations and electrophysiological recordings in awake monkeys. First, our simulations of a computational model of the striatal spiny neuron have confirmed the existence, in spike latency histograms of the earliest spikes, of two (early and late) peaks responding to excitatory cortical input, each of which coincides, respectively, with neuronal activity in the up or down state. Given that the membrane potential shifts of striatal spiny neurons were observed primarily in the anesthetized animal (Wilson and Groves, 1981; Wilson, 1993; Wilson and Kawaguchi, 1996;

This article is published in The Journal of Neuroscience, Rapid Communications Section, which publishes brief, peerreviewed papers online, not in print. Rapid Communications are posted online approximately one month earlier than they would appear if printed. They are listed in the Table of Contents of the next open issue of JNeurosci. Cite this article as: JNeurosci, 2002, 22:RC230 (1-6). The publication date is the date of posting online at www.jneurosci.org.

http://www.jneurosci.org/cgi/content/full/6482 
Stern et al., 1997, 1998; Wickens and Wilson, 1998), it still remains unclear whether those events may be relevant to changes in striatal neuron activity under awake conditions. In the second set of the present study, we extracellularly recorded, from behaving monkeys, the activity of striatal spiny neurons in response to electrical stimulation in the two cortical motor-related areas, the primary motor cortex (MI) and the supplementary motor area (SMA). To examine whether there exist two temporally separate peaks similar to those obtained from the numerical simulations, the earliest spike latency histograms (ESLHs) were constructed on the basis of the experimental data.

\section{MATERIALS AND METHODS}

Numerical simulations. Our model of the striatal spiny projection neuron consisted of two compartments, a soma (radius of $15 \mu \mathrm{m}$; length of 15 $\mu \mathrm{m})$ and a dendrite (radius of $15 \mu \mathrm{m}$; length of $400 \mu \mathrm{m}$ ) (Wickens and Arbuthnott, 1993; Kötter and Wickens, 1995). Membrane capacitance $\left(C_{\mathrm{m}}\right)$ of the neuron was given as $2 \mu \mathrm{F} / \mathrm{cm}^{2}$. Each compartment contained a leakage current, a spike-generating sodium current, and several types of potassium currents that characterize the membrane properties of the neuron. The kinetics of the sodium current were determined based on the mathematical description by Durstewitz et al. (2000); a voltage shift of $-8 \mathrm{mV}$ was made in the activation and inactivation functions to adjust spiking threshold. The maximum conductance $\left(g_{\mathrm{Na}}\right)$ was given as 120 $\mathrm{mS} / \mathrm{cm}^{2}$ for soma and $40 \mathrm{mS} / \mathrm{cm}^{2}$ for dendrite. In general, the striatal spiny neuron bears the following three voltage-dependent potassium currents that are responsible for the outward rectification at the depolarized up state: a fast inactivating A current (A current), a slowly inactivating A current (KS current), and a slow non-inactivating current (Surmeier et al., 1988; Nisenbaum et al., 1994; Nisenbaum and Wilson, 1995). The kinetics of the A current was determined according to Traub et al. (1991); a voltage shift of $-3 \mathrm{mV}$ was made in the activation and inactivation functions. The maximum conductance $\left(g_{\mathrm{A}}\right)$ was given as 3 $\mathrm{mS} / \mathrm{cm}^{2}$ for soma and $2 \mathrm{mS} / \mathrm{cm}^{2}$ for dendrite. The KS current was defined according to Wang (1993) $\left(g_{\mathrm{KS}}=0.2 \mathrm{mS} / \mathrm{cm}^{2}\right.$ for both soma and dendrite). The slow non-inactivating current was modeled as a potassium delayed rectifier current, of which activation and inactivation rates were divided by 18 on the basis of previous current-clamp analysis (Nisenbaum and Wilson, 1995). The maximum conductance $\left(g_{\text {non-inact }}\right)$ was given as $30 \mathrm{mS} / \mathrm{cm}^{2}$ for soma and $3 \mathrm{mS} / \mathrm{cm}^{2}$ for dendrite. In a hyperpolarized state, the striatal spiny neuron exhibits inward rectification through an anomalous potassium current (Uchimura et al., 1989). The voltage dependence of the anomalous potassium current was as described in a minimal model formulated by Nisenbaum and Wilson (1995) $\left(g_{\text {ir }}=\right.$ $0.4 \mathrm{mS} / \mathrm{cm}^{2}$ for both soma and dendrite). The equilibrium potentials were fixed as $E_{\text {leak }}=-75 \mathrm{mV}, E_{\mathrm{Na}}=50 \mathrm{mV}$, and $E_{\mathrm{K}}=-90 \mathrm{mV}$. The coupling constant between the somal and dendritic compartments was $0.29 \mu \mathrm{S}$.

It is well known that striatal spiny projection neurons are strongly innervated by excitatory glutamatergic input from the cerebral cortex. In the present simulation study, a single spiny projection neuron was presumed to receive 500 active corticostriatal afferent fibers that deliver Poisson spike trains constantly to its dendritic compartment $\left(g_{\mathrm{AMPA}}=\right.$ $0.33 \mathrm{nS}$ ). The synaptic currents followed first-order kinetic equations with single rate variables (Destexhe et al., 1998). An up and a down state were produced by changing the common firing rate of corticostriatal input. The firing rate was set as $\sim 40 \mathrm{~Hz}$ to achieve an up state (approximately $-60 \mathrm{mV}$ ) slightly below spike threshold, whereas the firing rate was set as $5 \mathrm{~Hz}$ to reach a down state (approximately $-80 \mathrm{mV}$ ) near the resting membrane potential. As the cortex was stimulated, the striatal spiny neuron received volleys that were mimicked by a Gaussian spike packet ( $\mathrm{SD}$ of $2 \mathrm{msec}$ ).

It is generally accepted that striatal interneurons exert a powerful inhibition on spiny projection neurons (Koos and Tepper, 1999). To examine the effects of the GABAergic feedforward inhibition on the activity of striatal projection neurons, we incorporated the $\mathrm{GABA}_{\mathrm{A}}$ receptor-mediated IPSPs in part of the simulations. The interneuron was modeled based on the Hodgkin-Huxley equation and stimulated by the same spike packet as given to the projection neuron. The equilibrium potential $\left(E_{\mathrm{GABA}}\right)$ was fixed as $-75 \mathrm{mV}$, and the maximum conductance $\left(g_{\mathrm{GABA}}\right)$ was set as $5 \mathrm{nS}$ to produce the amplitude of the observed IPSPs.

Electrophysiological recordings. Two female Japanese monkeys ( $\mathrm{Ma}$ - caca fuscata), weighing $5.0-6.0 \mathrm{~kg}$, were used for the present study. The experimental protocol was approved by the Animal Experiment Committee at the Tokyo Metropolitan Institute for Neuroscience, and all experiments were performed in line with the NIH Guide for the Care and Use of Laboratory Animals. First of all, the monkeys received a surgical operation to gain easy access to extracellular recording of striatal neuron activity in unanesthetized conditions. For intracortical implantation of stimulating electrodes, forelimb representations of the MI and SMA were identified under the guidance of intracortical microstimulation mapping. Pairs of bipolar electrodes (made of enamel-coated stainlesssteel wire with $200 \mu \mathrm{m}$ diameter; intertip distance, $2 \mathrm{~mm}$ ) were implanted chronically into the forelimb regions of the MI and SMA. Other technical details were as described previously (Nambu et al., 2000).

A glass-coated Elgiloy-alloy microelectrode $(0.5-1.5 \mathrm{M} \Omega$ at $1 \mathrm{kHz})$ was inserted obliquely ( $45^{\circ}$ from vertical in the frontal plane) into the putamen to record neuronal activity extracellularly. Investigations focused mainly on the caudal part of the putamen in which corticostriatal projections from the MI and SMA terminate densely (Takada et al., 1998). The unitary activity of putamen neurons was amplified $(8000 \times, 200-$ $2000 \mathrm{~Hz}$ ), converted to digital pulses with a window discriminator, and then fed to a computer. Electrical stimulation (300 $\mu \mathrm{sec}$ duration, single pulse; $0-0.8 \mathrm{~mA}$ ) was delivered in the MI and SMA through a constantcurrent stimulator at the fixed interstimulus interval of $1.3 \mathrm{sec}$. Responses of putamen neurons to the cortical stimulation with different intensities were observed and stored on a computer. The parameters of the window discriminator were carefully adjusted to avoid contamination by activities of other neurons, especially in the case of strong stimulation. The earliest spike elicited by every cortical stimulation was selected, and spike latency histograms (bin width, $0.5 \mathrm{msec}$; usually summed for 100 times) were constructed to obtain ESLHs. To provide solid evidence that dual peaks reflect distinct latencies, we fitted normalized ESLHs showing apparent dual peaks to dual Gaussian functions according to the Levenberg-Marquart method of nonlinear least squares. The model used was as follows:

$$
p(t)=\frac{\lambda}{\sqrt{2 \pi \sigma_{1}^{2}}} \exp \left(-\frac{\left(t-\tau_{1}\right)^{2}}{2 \sigma_{1}^{2}}\right)+\frac{1-\lambda}{\sqrt{2 \pi \sigma_{2}^{2}}} \exp \left(-\frac{\left(t-\tau_{2}\right)^{2}}{2 \sigma_{2}^{2}}\right),
$$

where $\tau_{1}$ and $\sigma_{1}\left(\tau_{2}\right.$ and $\left.\sigma_{2}\right)$ are the mean latency and the amplitude of timing fluctuations in the up (down) state, respectively, and $\lambda$ is the ratio of the up state to total.

The responses were also analyzed by constructing peristimulus time histograms (PSTHs) (bin width, $0.5 \mathrm{msec}$; usually summed for 100 times). To exclude any possibility that MI and SMA stimulation might induce a long-term potentiation or long-term depression in corticostriatal transmission (Charpier et al., 1999), the probability of spike firing and the latency of evoked spikes were compared among early, middle, and late trials, which were selected from 100 trials. Neither of them showed any signs of such synaptic plasticity. The ESLHs and PSTHs were smoothed through a Gaussian filter $(\tau=0.9 \mathrm{msec})$ (Szucs, 1998).

\section{RESULTS}

\section{Numerical simulations of striatal spiny neuron model}

First, we analyzed the behavior of a computational model of the striatal spiny neuron. In our model neuron, hyperpolarizing currents and subthreshold depolarizing currents induced inward or outward rectification, respectively (Fig. 1A). A suprathreshold current evoked action potentials with a prolonged delay of the earliest spike. It is most likely that the prolonged delay of action potential generation was ascribed to the outward rectification induced by the activation of the A current. Blockade of the sodium current by setting as $g_{\mathrm{Na}}=0 \mathrm{mS} / \mathrm{cm}^{2}$ for both the somal and dendritic compartments inhibited action potentials (Fig. 1B). Additional blockade of both the A and KS currents by setting as $g_{\mathrm{A}}=g_{\mathrm{KS}}=0 \mathrm{mS} / \mathrm{cm}^{2}$ induced a transient overshoot of the membrane potential in the early phase of step currents and outward rectification in the late phase (Fig. 1C). These events were well consistent with the changes in membrane potential of striatal spiny neurons after application of tetrodotoxin (sodium channel blocker) and 4-aminopyridine (A and KS current blocker at high concentrations). Thus, the behavior of the striatal spiny 


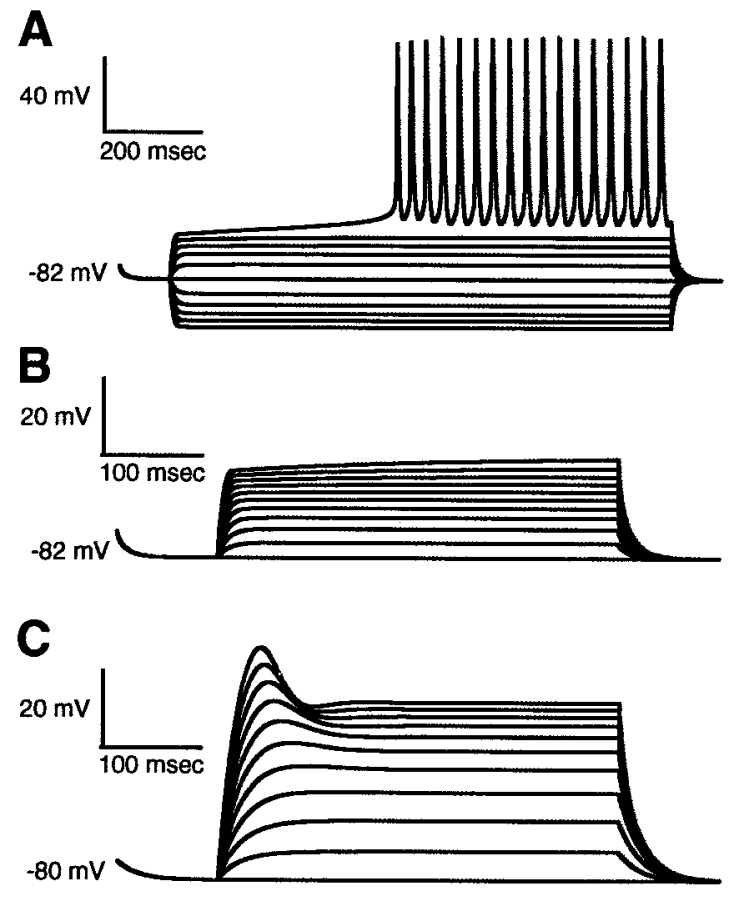

Figure 1. Membrane potentials of the striatal spiny neuron model. $A$, Responses when hyperpolarizing and depolarizing currents were injected. $B$, Responses when $g_{\mathrm{Na}}$ was set as $0 \mathrm{mS} / \mathrm{cm}^{2}$. C, Responses when $g_{\mathrm{Na}}, g_{\mathrm{A}}$, and $g_{\mathrm{KS}}$ were set as $0 \mathrm{mS} / \mathrm{cm}^{2}$.

neuron model constructed in the present study could highly simulate the experimental results of the in vitro intracellular recording study of Nisenbaum and Wilson (1995).

A Gaussian spike packet that mimicked excitatory cortical input evoked action potentials in the model neuron with a short latency when the neuron was initially in the up state (Fig. 2A). Given a long synaptic integration time required for a weak input, the latency became longer as the input was weaker. Only sufficiently strong inputs could elicit spikes in the model neuron that was initially set in the down state (Fig. $2 A$, asterisks). If repetitive transitions between the two states occur with approximately equal probabilities of visit, in the striatal spiny neuron, then the ESLH of neuronal activity evoked by cortical input should resemble a sum of the two histograms calculated separately for each state. In fact, the summed ESLHs obtained from numerical simulations of the model neuron displayed dual peaks for stronger inputs and single peaks for weaker inputs (Fig. 2A). Moreover, we incorporated the effects of GABAergic feedforward inhibition on the activity of the striatal spiny neuron model (Fig. 2B). The excitatory cortical input elicited two or three spikes in a GABAergic interneuron. In the up state, the onset of an IPSC did not precede the generation of an action potential in a spiny projection neuron (Fig. 2C). On the other hand, the IPSC occurred a little earlier than the action potential in the down state and, consequently, delayed it by a few milliseconds. Thus, except that the latency was slightly prolonged in the down state (Fig. $2 A, B$, asterisks), the patterns of ESLHs were almost identical to those of the histograms calculated in the absence of the GABAergic inhibition.

\section{Electrophysiological recordings in awake monkeys}

The results from our numerical simulations indicate that ESLHs of the striatal spiny neuron model exhibit two temporally distinct peaks in response to excitatory cortical input. In the second set of
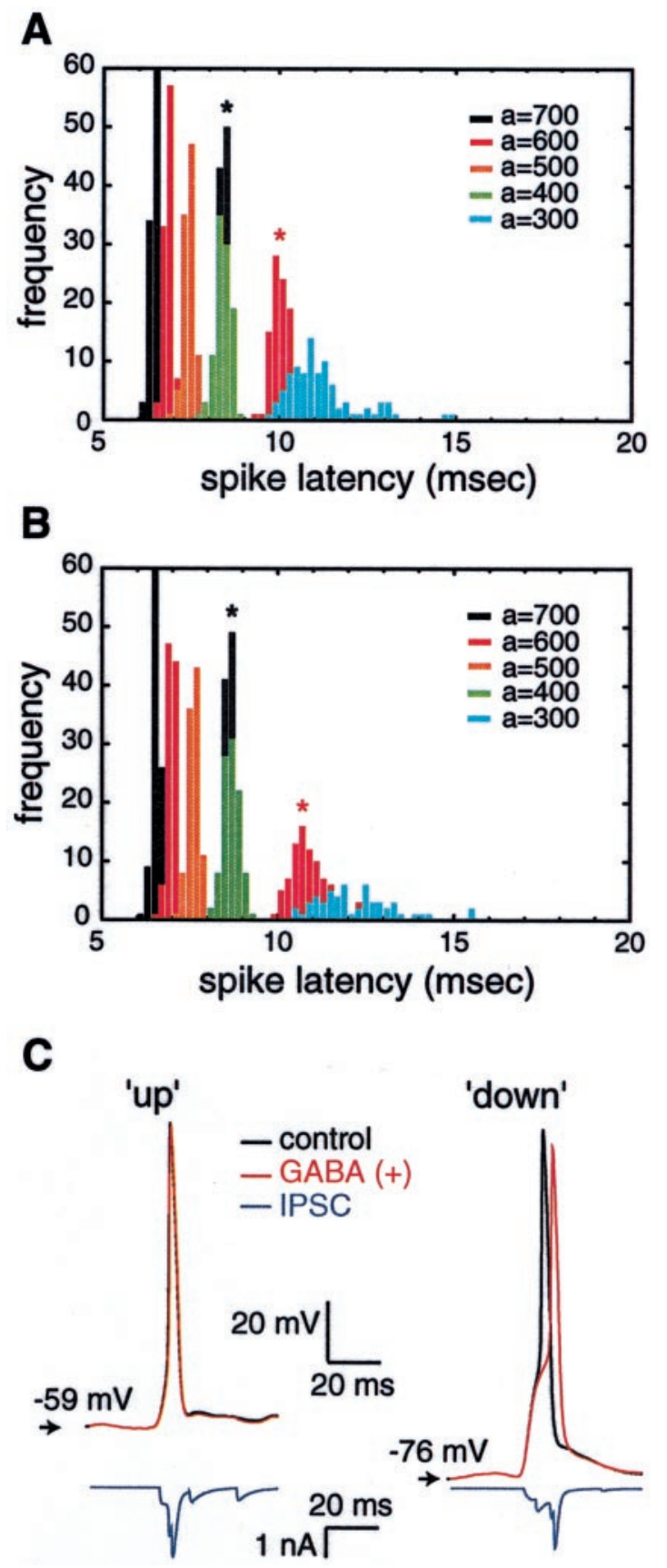

Figure 2. ESLHs and response profiles of the striatal spiny neuron model. $A$, ESLHs calculated for synaptic inputs of different intensities (represented by different colors). Each intensity is defined as the number of spikes $(a)$ in a packet. $B$, ESLHs when GABAergic feedforward inhibition was incorporated. In $A$ and $B$, asterisks denote latency distributions of the model neuron that was initially set in the down state. $C$, Response profiles of the model neuron with (red) and without (black) GABAergic feedforward inhibition. In the left or right traces, the neuron was initially set in the up or down state, respectively. The IPSC (blue) in each case was also displayed.

the present study, we examined whether similar phenomena might be observed experimentally in striatal neurons under awake conditions. Thus, identified regions of the MI and SMA representing the forelimb were electrically stimulated in behaving monkeys, and unitary activities responding to the cortical stimulation were extracellularly recorded in the putamen. 
Based on the patterns of spontaneous activities, striatal neurons can be classified into two groups: phasically active neurons (PANs), which are silent at rest and are activated phasically during voluntary movement, and tonically active neurons (TANs), which have tonic background discharges at $2-10 \mathrm{~Hz}$ and action potentials with long durations (Alexander and DeLong, 1985; Aosaki et al., 1994, 1995). It has been considered that PANs and TANs correspond to medium-sized spiny projection neurons and large aspiny cholinergic interneurons, respectively. In the present experiments, we analyzed a total of 24 putamen neurons (23 PANs and one TAN) that displayed excitatory responses to electrical stimulation in the MI and SMA. Of 23 PANs examined, 21 neurons had two (i.e., early and late) peaks in their ESLHs (Table 1). A typical example of PAN activity in response to MI stimulation is shown in Figure $3 A$. Single cortical stimulation evoked repetitive firing consisting of several spikes. The earliest spike elicited by every stimulation (Fig. $3 A_{1}$, filled circles) was collected, and ESLHs were constructed (Fig. $3 A_{2}$ ). When the stimulus intensity was weak $(<0.65 \mathrm{~mA})$, no dual peaks were clearly detected in ESLHs. As the stimulus intensity increased $(>0.75 \mathrm{~mA})$, there sharply appeared two temporally separate peaks. For a stimulus intensity of $0.8 \mathrm{~mA}$, the ESLH was fitted to a dual Gaussian function with $\lambda=0.36, \tau_{1}=114.4 \mathrm{msec}, \sigma_{1}=0.5$ msec, $\tau_{2}=118.5$, and $\sigma_{2}=1.8 \mathrm{msec}$ (Fig. $3 A_{2}$, inset) (for a stimulus intensity of $0.75 \mathrm{~mA}, \lambda=0.24, \tau_{1}=114.7 \mathrm{msec}, \sigma_{1}=$ $0.57 \mathrm{msec}, \tau_{2}=119.3$, and $\sigma_{2}=1.7 \mathrm{msec}$ ). Similar peaks to those seen in the ESLHs were also observed in PSTHs of the same neuron (Fig. $3 A_{3}$ ). A typical example of PANs responding to SMA stimulation is depicted in Figure $3 B$. In ESLHs of this neuron, the relationship between the stimulus intensity and the wave pattern was essentially the same as in those of PANs responding to MI stimulation, although early peaks were usually small (Fig. $3 B_{2}$ ). A dual Gaussian fitting of the ESLH for a stimulus intensity of $0.38 \mathrm{~mA}$ was obtained with $\lambda=0.36, \tau_{1}=$ $111.6 \mathrm{msec}, \sigma_{1}=0.74 \mathrm{msec}, \tau_{2}=115.0 \mathrm{msec}$, and $\sigma_{2}=0.84 \mathrm{msec}$ (Fig. $3 B_{2}$, inset). A small early peak that was likely to coincide with the early peak in the ESLH was found in the PSTH of the same neuron (Fig. $3 B_{3}$, arrowhead). The time intervals between the early and late peaks in the ESLHs were $3.8 \pm 1.0$ or $4.1 \pm 0.8$ msec for MI or SMA stimulation, respectively. We also analyzed one TAN responding to SMA stimulation. However, no dual peaks were seen in ESLHs, although the stimulus intensity increased as strong as $0.7 \mathrm{~mA}$ (Table 1).

\section{DISCUSSION}

Given the bistability of the membrane potential in striatal spiny neurons, their spike latency in the up state must be shorter than that in the down state. Based on this dual-latency hypothesis, we analyzed the cortically induced two-state membrane potential transitions from both computational and electrophysiological viewpoints. In our numerical simulations of a striatal spiny neu-

\begin{tabular}{|c|c|c|}
\hline & PANs & TANs \\
\hline MI & 17 (17) & $0(0)$ \\
\hline SMA & $4(6)$ & $0(1)$ \\
\hline Total & $21(23)$ & $0(1)$ \\
\hline
\end{tabular}

$\overline{\text { Each numeral in parentheses represents the number of neurons showing excitatory }}$ responses to cortical stimulation. ron model, the existence of two (early and late) peaks has been shown in ESLHs of neuronal activity evoked by excitatory cortical input. Consistent with the results of the simulation study, the present extracellular unit recordings in awake monkeys have elucidated that most of the PANs receiving input from the MI and SMA typically display two temporally separate peaks in their ESLHs. Thus, it can be interpreted that the early and late peaks in response to stronger inputs (or stimuli) probably correspond to spikes elicited in the up or down state, respectively. The spikes in the down state may entail a transition to the up state, during which striatal spiny neurons can fire.

Otherwise, an incoming volley itself might have two peaks; the early peak is derived directly from the stimulated cortical area, whereas the late peak is derived indirectly, for example, from another cortical area connected with the stimulated area. In this case, however, strong stimulation will create single early peaks in ESLHs, given that it can almost always activate so many neurons in the stimulated cortical area as to elicit the earliest spikes in PANs through the direct corticostriatal projection. This is obviously contradictory to the present data, and, therefore, such an alternative interpretation of the dual peaks in spike latency distribution seems unlikely.

The spike latency distributions obtained in our study were broader in the experiments than in the simulations. This is presumably because the membrane potential of striatal spiny neurons may actually take various values between the up and down states, particularly during down transitions from the up to the down state, although it was clamped at either the up or down state in the simulations. Also, the narrow Gaussian spike packet adopted in our simulations could only generate such narrow distributions of spike latency. The probabilities of the up and down states may give a free parameter in constructing ESLHs based on simulation data and need not be equal. However, the present results imply that the probabilities are not too far off equality.

In the present numerical simulations, inhibitory input derived from striatal GABAergic interneurons did not affect the early peaks (in the up state) in ESLHs but did slightly extend the latency for the late peaks (in the down state) (Fig. $2 A, B$ ). Because activation of the GABAergic synaptic input to striatal spiny neurons nearly coincides with their spike generation in the up state (Fig. $2 C$ ), the timing of the early peaks is not significantly altered by the input. In the down state, on the other hand, the activated GABAergic input precedes the spike generation in striatal spiny neurons, to delay it by only a few milliseconds (Fig. $2 C)$. Thus, although GABAergic feedforward inhibition makes the spike latency for the late peaks a little longer, it still rarely prevents the spike generation itself.

In vivo intracellular recordings performed in anesthetized rats consistently showed that cortical stimulation could evoke shortlatency EPSPs, followed by a prolonged hyperpolarization $(\sim 400$ msec) resembling the up state (Wilson, 1993). When the cortex was stimulated in awake monkeys, similar increased firing was indeed induced at latencies comparable with the late evoked up state (Nambu et al., 2002). This would be an additional piece of evidence suggesting the presence of the up and down states in awake monkeys. In our study, $\sim 20 \%$ of the recorded PANs increased their spike firing at latencies of 300-400 msec.

In our electrophysiological recordings, a few of the identified PANs, especially those with SMA input, had only single peaks in their ESLHs. The occurrence of single peaks that are likely to represent spikes elicited in the up state might be explained by 
$A_{1}$

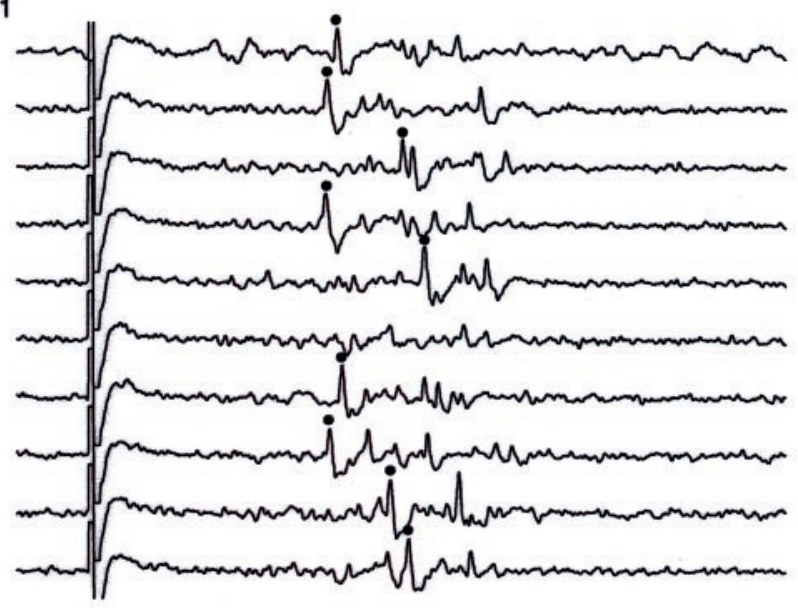

$\uparrow$

Ml stim $0.8 \mathrm{~mA}$

$\mathrm{A}_{2}$

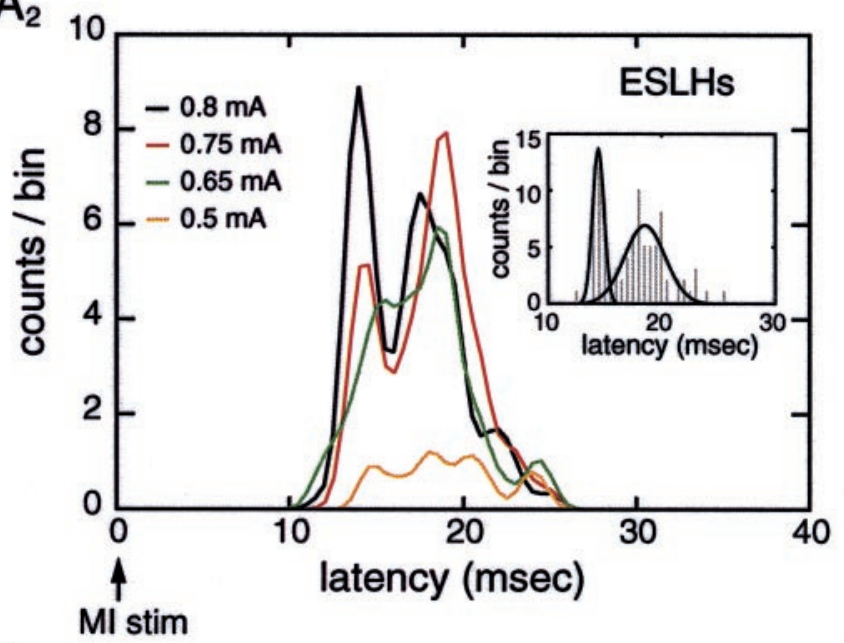

$\mathrm{A}_{3}$

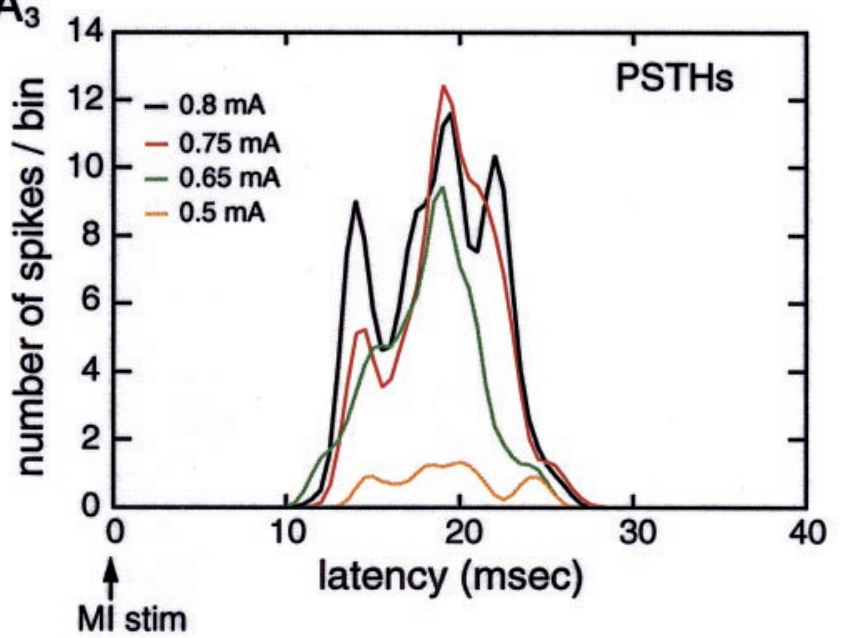

B.

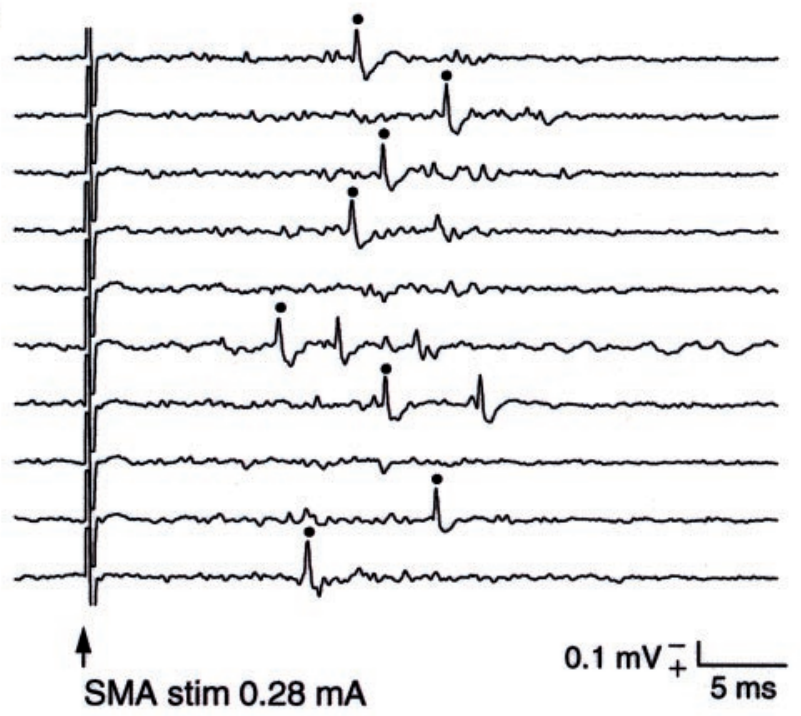

$\mathrm{B}_{2}$

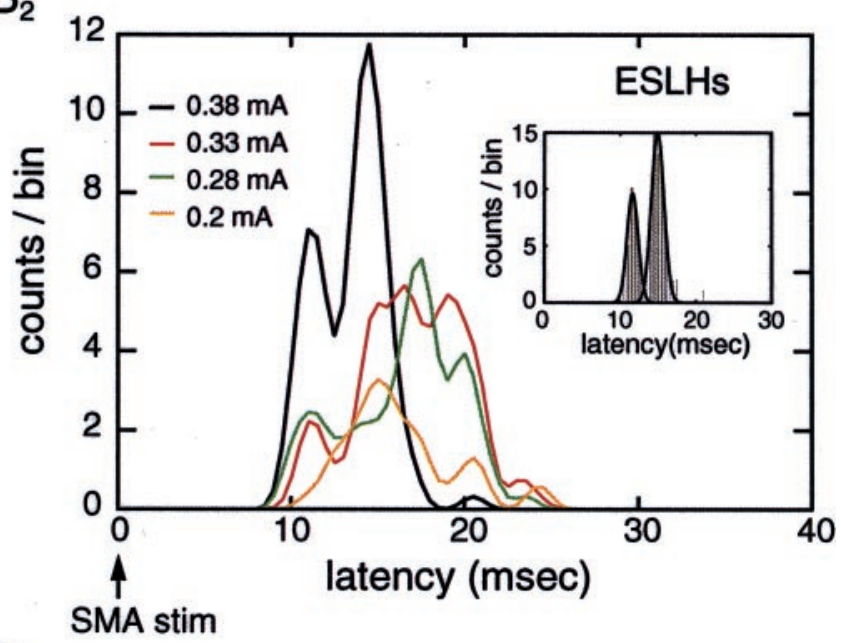

$\mathrm{B}_{3}$

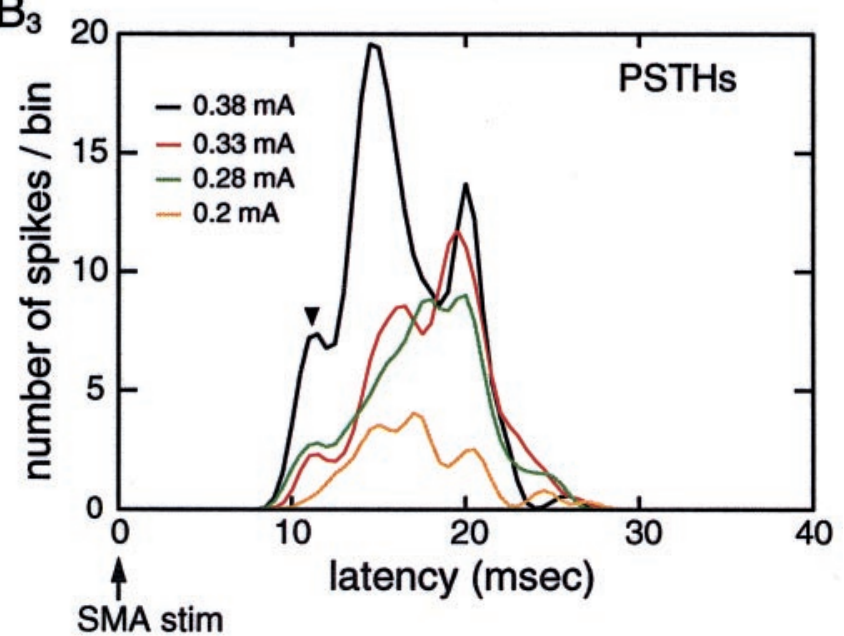

Figure 3. Data obtained from extracellular unit recordings. $A$, PAN receiving input from the MI. $A_{1}$, Raw data of 10 consecutive trials selected from 100 trials. The forelimb region of the MI was stimulated (MI stim) at $0.8 \mathrm{~mA}$. The earliest spike in each trial (specified by filled circles) was collected, and ESLHs were constructed. $A_{2}, A_{3}$, ESLHs (bin width, $0.5 \mathrm{msec}$ ) and PSTHs (bin width, $0.5 \mathrm{msec}$ ). Stimulation in the MI (MI stim) was performed with different intensities of currents (represented by different colors) at time 0 (arrow), and neuronal responses were summed 100 times for each intensity. Inset in $A_{2}$, Dual Gaussian fitting of the ESLH for a stimulus intensity of $0.8 \mathrm{~mA}$. $B$, PAN receiving input from the SMA. $B_{1}$, Raw data of 10 successive trials with a stimulus intensity of $0.28 \mathrm{~mA}$. Calibration in $B_{1}$ is also applicable to $A_{1} . B_{2}, B_{3}$, ESLHs and PSTHs. Inset in $B_{2}$, Dual Gaussian fitting of the ESLH for a stimulus intensity of $0.38 \mathrm{~mA}$. Other conventions are as in $A_{1}-A_{3}$. Single stimulation in the MI and SMA elicited several repetitive spikes in PANs $\left(A_{1}, B_{1}\right)$, especially in the case of high stimulus intensity, and, therefore, the later component of the repetitive spikes occasionally formed a third peak in PSTHs $\left(A_{3}, B_{3}\right)$. 
postulating that the up and down states are so far apart that the cortical stimulation can evoke responses only in the up state. In fact, it has been shown that medium spiny neurons in the nucleus accumbens fire in response to prefrontal cortical stimulation only during the up state (O'Donnell and Grace, 1995). Another possible but opposite explanation is that the difference in membrane potential level between the up and down states is not large enough to detect dual peaks. With respect to TANs, the activity of only one TAN was recorded in our experiments, and there existed single peaks in its ESLHs. This favors previous data that TANs do not appear to possess the two distinct states of the membrane potential (Wilson et al., 1990; Wilson, 1993).

The activity of spiny projection neurons in the striatum has been implicated in a variety of motor behavior, including motor learning (Graybiel et al., 1994; Graybiel, 1995). The present results suggest that striatal spiny neurons of awake monkeys may probably exhibit the membrane potential shifts between the up and down states, as revealed in anesthetized rats (Wilson and Groves, 1981; Wilson, 1993; Wilson and Kawaguchi, 1996; Stern et al., 1997, 1998; Wickens and Wilson, 1998). The patterns of spike firing of striatal spiny neurons are considered to be a reflection of convergent and perhaps synchronous input arising from the cortex. The spontaneous subthreshold membrane potential fluctuations similar to those observed in striatal spiny neurons have been shown recently to occur in corticostriatal neurons (Cowan and Wilson, 1994; Wilson, 1995; Stern et al., 1997). It has also been reported that the two-state membrane potential transitions are highly correlated among striatal spiny neurons (Stern et al., 1998). The same approach that we took here may be applicable to exploring the distributions of the up and down states during motor behavior over a population of spiny projection neurons (Câteau and Fukai, 2001). Thus, to clarify the functional role of such synchronized membrane potential state transitions, it is of interest to analyze the activity of both corticostriatal and striatal spiny neurons during the performance of a motor task.

\section{REFERENCES}

Alexander GE, DeLong MR (1985) Microstimulation of the primate neostriatum. II. Somatotopic organization of striatal microexcitable zones and their relation to neuronal response properties. J Neurophysiol 53:1417-1430.

Aosaki T, Tsubokawa H, Ishida A, Watanabe K, Graybiel AM, Kimura M (1994) Responses of tonically active neurons in the primate's striatum undergo systematic changes during behavioral sensorimotor conditioning. J Neurosci 14:3969-3984.

Aosaki T, Kimura M, Graybiel AM (1995) Temporal and spatial characteristics of tonically active neurons of the primate's striatum. J Neurophysiol 73:1234-1252.

Câteau H, Fukai T (2001) Fokker-Planck approach to the pulse packet propagation in synfire chain. Neural Networks 14:675-685.

Charpier S, Mahon S, Deniau J-M (1999) In vivo induction of striatal long-term potentiation by low-frequency stimulation of the cerebral cortex. Neuroscience 91:1209-1222.

Cowan RL, Wilson CJ (1994) Spontaneous firing patterns and axonal projections of single corticostriatal neurons in the rat medial agranular cortex. J Neurophysiol 71:17-32.

Destexhe A, Mainen ZF, Sejnowski TJ (1998) Kinetic models of synaptic transmission. In: Methods in neural modeling (Koch C, Segev I, eds), pp 1-25. Cambridge, MA: MIT.

Durstewitz D, Seamans JK, Sejnowski TJ (2000) Dopamine-mediated stabilization of delay-period activity in a network model of prefrontal cortex. J Neurophysiol 83:1733-1750.
Graybiel AM (1995) Building action repertoires: memory and learning functions of the basal ganglia. Curr Opin Neurobiol 5:733-741.

Graybiel AM, Aosaki T, Flaherty AW, Kimura M (1994) The basal ganglia and adaptive motor control. Science 265:1826-1831.

Kawaguchi Y, Wilson CJ, Emson PC (1989) Intracellular recording of identified neostriatal patch and matrix spiny cells in a slice preparation preserving cortical inputs. J Neurophysiol 62:1052-1068.

Koos T, Tepper JM (1999) Inhibitory control of neostriatal projection neurons by GABAergic interneurons. Nat Neurosci 2:467-472.

Kötter R, Wickens J (1995) Interactions of glutamate and dopamine in a computational model of the striatum. J Comput Neurosci 2:195-214.

Nambu A, Tokuno H, Hamada I, Kita H, Imanishi M, Akazawa T, Ikeuchi Y, Hasegawa N (2000) Excitatory cortical inputs to pallidal neurons via the subthalamic nucleus in the monkey. J Neurophysiol 84:289-300.

Nambu A, Kaneda K, Tokuno H, Takada M (2002) Organization of corticostriatal motor inputs in monkey putamen. J Neurophysiol, in press.

Nisenbaum ES, Wilson CJ (1995) Potassium currents responsible for inward and outward rectification in rat neostriatal spiny projection neurons. J Neurosci 15:4449-4463.

Nisenbaum ES, Xu ZC, Wilson CJ (1994) Contribution of a slowly inactivating potassium current to the transition to firing of neostriatal spiny projection neurons. J Neurophysiol 71:1174-1189.

O'Donnell P, Grace AA (1995) Synaptic interactions among excitatory afferents to nucleus accumbens neurons: hippocampal gating of prefrontal cortical input. J Neurosci 15:3622-3639.

Parent A, Hazrati L-N (1995) Functional anatomy of the basal ganglia. I. The cortico-basal ganglia-thalamo-cortical loop. Brain Res Rev 20:91-127.

Stern EA, Kincaid AE, Wilson CJ (1997) Spontaneous subthreshold membrane potential fluctuations and action potential variability of rat corticostriatal and striatal neurons in vivo. J Neurophysiol 77:1697-1715.

Stern EA, Jaeger D, Wilson CJ (1998) Membrane potential synchrony of simultaneously recorded striatal spiny neurons in vivo. Nature 394:475-478.

Surmeier DJ, Bargas J, Kitai ST (1988) Voltage-clamp analysis of a transient potassium current in rat neostriatal neurons. Brain Res 473:187-192.

Szucs A (1998) Applications of the spike density function in analysis of neuronal firing patterns. J Neurosci Methods 81:159-167.

Takada M, Tokuno H, Nambu A, Inase M (1998) Corticostriatal projections from the somatic motor areas of the frontal cortex in the macaque monkey: segregation versus overlap of input zones from the primary motor cortex, the supplementary motor area, and the premotor cortex. Exp Brain Res 120:114-128.

Traub RD, Wong RK, Miles R, Michelson H (1991) A model of a CA3 hippocampal pyramidal neuron incorporating voltage-clamp data on intrinsic conductances. J Neurophysiol 66:635-650.

Uchimura N, Cherubini E, North RA (1989) Inward rectification in rat nucleus accumbens neurons. J Neurophysiol 62:1280-1286.

Wang XJ (1993) Ionic basis for intrinsic $40 \mathrm{~Hz}$ neuronal oscillations. NeuroReport 5:221-224.

Wickens JR, Arbuthnott GW (1993) The corticostriatal system on computer simulation: an intermediate mechanism for sequencing of actions. Prog Brain Res 99:325-339.

Wickens JR, Wilson CJ (1998) Regulation of action-potential firing in spiny neurons of the rat neostriatum in vivo. J Neurophysiol 79:2358-2364.

Wilson CJ (1993) The generation of natural firing patterns in neostriatal neurons. Prog Brain Res 99:277-297.

Wilson CJ (1995) The contribution of cortical neurons to the firing pattern of striatal spiny neurons. In: Models of information processing in the basal ganglia (Houk JC, Davis JL, Beiser DG, eds), pp 29-50. Cambridge, MA: MIT

Wilson CJ, Groves PM (1981) Spontaneous firing patterns of identified spiny neurons in the rat neostriatum. Brain Res 220:67-80.

Wilson CJ, Kawaguchi Y (1996) The origins of two-state spontaneous membrane potential fluctuations of neostriatal spiny neurons. J Neurosci 16:2397-2410.

Wilson CJ, Chang HT, Kitai ST (1983) Disfacilitation and long-lasting inhibition of neostriatal neurons in the rat. Exp Brain Res 51:227-235.

Wilson CJ, Chang HT, Kitai ST (1990) Firing patterns and synaptic potentials of identified giant aspiny interneurons in the rat neostriatum. J Neurosci 10:508-519. 